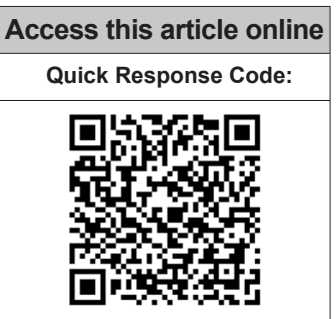

Website:

www.jponline.org

DOI:

10.4103/JLP.JLP_116_18
Department of Microbiology, Hi-Tech Medical College and Hospital, Rourkela, ${ }^{1}$ Department of Skin and

VD, SCB Medical College and Hospital, Cuttack,

Odisha, India

Address for correspondence: Dr. Shreekant Tiwari,

S. O. Qr. No. 1 SCB Campus, Ranihat Medical Road, Cuttack - 753 007, Odisha, India.

E-mail: drshreekant@ rediffmail.com

Submission: 06-09-2018 Accepted: 12-11-2018

\title{
Bacteremia caused by Comamonas testosteroni an unusual pathogen
}

\author{
Shreekant Tiwari, Monalisah Nanda ${ }^{1}$
}

\section{Abstract:}

Comamonas species are rare isolates in microbiology laboratories and have been infrequently reported as an infectious agent in routine clinical practice. They have a wide range of natural habitats including water, soil, and plants as well as from some hospital devices, such as intravenous lines and the reservoir water in the humidifiers of respiratory therapy equipment. Comamonas testosteroni is rarely recognized as a human pathogen. In spite of its uncommon human pathogenesis, there are few reports where it was reported as an aggressive opportunistic pathogen, and that was mostly related to Testosterone species. Herewith, we are reporting this pathogen from the blood of an immunocompetent female. The aim of this case report is to alert clinicians and laboratory physicians for the potential diagnosis and clinical approach of bloodstream infections caused by such unusual pathogens. This is the first documented case of bacteremia caused by $C$. testosteroni from India.

Key words:

Bacteremia, Comamonas testosteroni, immunocompetent

\section{Introduction}

Comara omamonas testosteroni is an aerobic Gram-negative bacillus that is oxidase positive, motile, nonspore forming, nonglucose fermenting, and arranged either singly or in pairs. ${ }^{[1]}$ It was formerly known as Pseudomonas testosteroni, and now this bacillus was reclassified by molecular genetics. It was renamed as $C$. testosteroni as it is known to use testosterone as the sole source of carbon for their growth. Although it has low virulence potency, however, there are few case reports on its involvement in human infections. became clinically important after 1987 when reports began accumulating on human infections such as peritonitis, cellulitis, endocarditis, meningitis, pneumonia, endophthalmitis, and tenosynovitis ${ }^{[2]}$ but cases of bloodstream infections caused by $C$. testosteroni have been infrequently reported. C. testosteroni is commonly found

This is an open access journal, and articles are distributed under the terms of the Creative Commons Attribution-NonCommercial-ShareAlike 4.0 License, which allows others to remix, tweak, and build upon the work non-commercially, as long as appropriate credit is given and the new creations are licensed under the identical terms.

For reprints contact: reprints@medknow.com in soil, water saprophytes, and plants. It has also been detected in the water of in-use hospital oxygen humidifier reservoirs, hemodialysis solutions, urine, mucus, prostate tissue, stool, and respiratory secretions of cystic fibrosis patients. ${ }^{[3]}$ However, it has not been recognized as a component of the endogenous human microflora. The genus Comamonas originally contained following species: Comamonas terrigena, C. testosteroni, Comamonas denitrificans Comamonas nitrativorans, and Comamonas kerstersii. It now contains 17 species, while Comamonas acidovorans has been separated from the genus on the basis of its 16S rRNA gene and is now known as Delftia acidovorans. Although pathogenic potential of $C$. testosteroni has not been studied well, it is usually considered as a commensals. Around 36 cases have been reported in medical literature concerning C. testosteroni infection so far. ${ }^{[3]}$ Herewith, we are reporting the first documented case of C. testosteroni bloodstream infection from India.

How to cite this article: Tiwari S, Nanda M. Bacteremia caused by Comamonas testosteroni an unusual pathogen. J Lab Physicians 2019;11:87-90. 


\section{Case Report}

A 46-year-old female from rural area admitted to our hospital with chief complains of high-grade fever $\left(103^{\circ} \mathrm{F}\right)$, headache, fast breathing, and burning micturition for the past 6 days. On examination, other findings were such as respiratory rate $82 / \mathrm{min}$, bilateral fine crepitation, pulse rate $146 / \mathrm{min}$, and blood pressure $140 / 90 \mathrm{mmHg}$. Central nervous system, cardiovascular system, and per abdominal examinations were within normal limit. Other relevant laboratory findings include hemoglobin $10 \mathrm{gm} \%$, total white blood cell count $24,200 / \mathrm{cmm}$, neutrophil $90 \%$, lymphocyte $10 \%$, fasting blood sugar $-88 \mathrm{mg} / \mathrm{dl}$, erythrocyte sedimentation rate $50 \mathrm{~mm}$ after $1 \mathrm{~h}, \mathrm{Na}-123 \mathrm{~m}$. mol/L, K $-4.2 \mathrm{~m} . \mathrm{mol} / \mathrm{L}$, blood urea $-45 \mathrm{mg} / \mathrm{dl}$, serum creatinine $-1.8 \mathrm{mg} / \mathrm{dl}$, urine routine microscopy shows pus cells $6-10 / \mathrm{hpf}$, albumin-trace, and no parasites. Serological tests such as $\mathrm{HBsAg}$, HIV, HCV, and dengue (NS1Ag, IgM, and IgG) were negative by Enzyme-linked immunosorbent assay. Chest X-ray shows bilateral opacities in lower lobe and ultrasound sonography (USG) abdomen was normal. Blood was collected with complete aseptic precaution in aerobic and anaerobic blood culture bottle (BacT/ALERT/3D; bioMerieux, Marcy l'Etoile, France). The patient was then started empirically with piperacillin + tazobactam and vancomycin. Aerobic blood culture bottle showed a positive sign of growth after $72 \mathrm{~h}$. The broth was then subcultured on 5\% sheep blood agar and MacConkey agar. After overnight incubation, MacConkey agar showed nonlactose fermenting colony but colony morphology was better seen after $48 \mathrm{~h}$ of aerobic incubation. Colonies were 1-2 mm in diameter, translucent, smooth surface, and nonpigmented [Figure 1]. Blood agar plate showed similar colony morphology and nonpigmented. Gram-stain was done from both the culture plates, and it was Gram-negative bacilli. It was motile by hanging

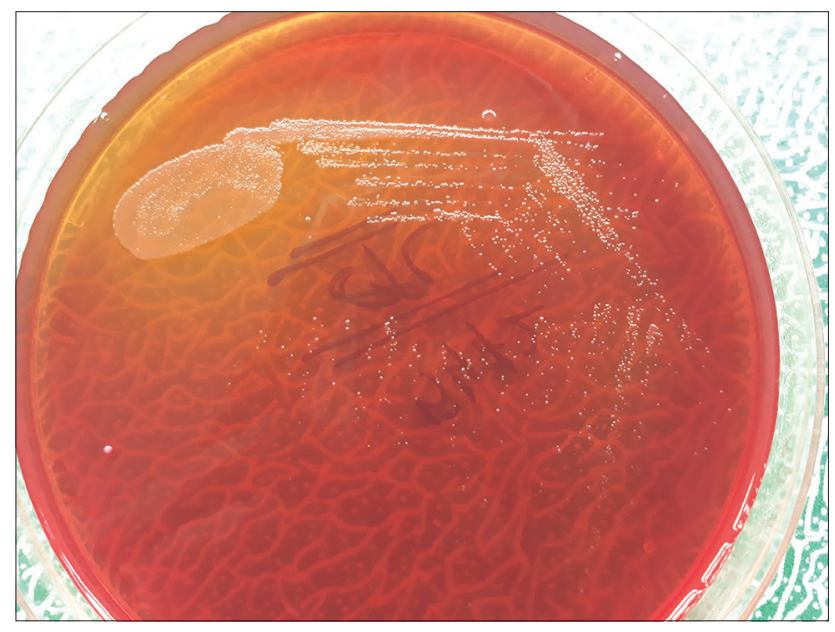

Figure 1: Nonlactose fermenting colony of Comamonas testosteroni on MacConkey agar drop method. Routine biochemical tests were done and it was catalase positive, oxidase positive, indole not produced, urea not hydrolyzed, citrate not utilized, TSI medium showed both slant, and butt alkaline without $\mathrm{H}_{2} \mathrm{~S}$ production. There was no hemolysis on blood agar culture plate. Further identification was done by VITEK-2 (fully automated identification system) using GN-25 card (bioMerieux, Marcy l'Etoile France). It was identified as C. testosteroni with $98 \%$ probability. There was no growth in anaerobic blood culture bottle even after 7 days of anaerobic incubation. Urine sample was also sent to the microbiology department for culture and sensitivity. It was processed on Cysteine lactose electrolyte deficient media and similar colony morphology was seen as on MacConkey and blood agar. Further identification was done by VITEK-2 using GN-25 card, and again it was identified as $C$. testosteroni with $97 \%$ probability. The isolation of same bacteria from both the clinical samples (blood and urine) confirmed its pathogenic role. The isolate was found to be sensitive to gentamicin, amikacin, imipenem, meropenem, ciprofloxacin, tigecycline, cotrimoxazole, cefuroxime, colistin, and resistant to piperacilline-tazobactam. Therapy was changed to gentamicin $4 \mathrm{mg} / \mathrm{kg} /$ dose daily and imipenem $25 \mathrm{mg} / \mathrm{kg} /$ dose 8 hourly for 10 days. The patient responded well to antibiotic therapy. Fever was subsided $3^{\text {rd }}$ day after starting therapy. Blood sample was taken again for culture, 7 days after completion of antibiotic therapy, and it was negative. Urine sample was also collected with complete aseptic precaution for culture and there was no growth. The patient is doing well during follow-up.

\section{Discussion}

C. testosteroni is a widely distributed aerobic Gram-negative bacillus which is rarely seen as a human pathogen. Now a day, this bacterium has been identified in various hospital devices; however, it has not been recognized as a component of the endogenous human microflora. Because this bacterium has been more frequently encountered (till now around 36 cases), we thought that this case would be beneficial for clinicians and laboratory physicians in their diagnosis and clinical approach $^{[4]}$ [Table 1]. Majority of the infections are community-acquired rather than nosocomial. ${ }^{[5,6]}$ Most of the previously reported patients were immunodeficient due to malignancy, chronic liver disease, and end-stage renal disease taking hemodialysis. ${ }^{[7,8]}$ However, in our case, the patient was immunocompetent based on the results of their previous reports. C. testosteroni is the most common species among its various species causing human infection and majority of the patients were survived. Till now around 16 cases of $C$. testosteroni bacteremia reported in medical literature, and out of which majority were male but our case 
Table 1: Comamonas testosteroni infections in the medical literature

\begin{tabular}{|c|c|c|c|c|c|}
\hline Age/sex & Diagnosis & Sample & Antibiotic treatment & Result & References \\
\hline 54 years/female & Catheter related infection & Blood & Cefepime, ciprofloxacin & Recovered & Abraham et al. \\
\hline 50 years/male & Cholesteatoma & CSF & Meropenem & Recovered & Arda et al. \\
\hline 31 years/female & Bacteremia & Bone marrow & Kanamycin, tetracycline & Recovered & Atkinson et al. \\
\hline 31 years/male & Appendicitis & Abscess & Cefoxitin & Recovered & Barbaro et al. \\
\hline 24 years/female & IVDA & CSF & Moxalactam and nafcillin & Recovered & Barbaro et al. \\
\hline 59 year/female & Alcoholic cirrhosis & Peritoneal fluid & Cefoxitin & Recovered & Barbaro et al. \\
\hline 11 years/male & Appendicitis & Peritoneal fluid & $\begin{array}{l}\text { Ampicillin, clindamycin, } \\
\text { tobramycin }\end{array}$ & Recovered & Barbaro et al. \\
\hline 12 years/female & Appendicitis & Peritoneal fluid & Cefoxitin & Recovered & Barbaro et al. \\
\hline 21 years/female & Appendicitis & Peritoneal fluid & Cefoxitin & Recovered & Barbaro et al. \\
\hline NB/Stillbirth & Maternal IVDA & Umblical cord-blood & - & Stillbirth & Barbaro et al. \\
\hline 84 years/female & $\mathrm{CHF}$ & Urine & Ampicillin & Recovered & Barbaro et al. \\
\hline 24 years/male & Appendicitis & Peritoneal fluid & Cefoxitin & Recovered & Barbaro et al. \\
\hline Female/NB & Maternal IVDA & Blood & Ampicillin, amikacin & Dead & Barbaro et al. \\
\hline 49 years/male & Infective endocarditis & Blood and mitral valve & Cefepime, gentamicin & Recovered & Cooper et al. \\
\hline No data & AIDS & Respiratory secretions & Ceftazidime & Recovered & Franzetti et al. \\
\hline 22 years/male & Appendicitis & Peritoneal fluid, blood & Cefazolin & Recovered & Gul M et al. \\
\hline 75 years/female & Catheter related infection & Blood and CVC & Ceftazidime, gentamicin & Recovered & Le Moal et al. \\
\hline 54 years/male & Cellulitis & Blood & Oxacillin, ciprofloxacin & Recovered & Tsui et al. \\
\hline 73 years/male & $\begin{array}{l}\text { Intra-abdominal infection and } \\
\text { hepatocellular carcinoma }\end{array}$ & Blood & Gentamicin, levofloxacin & Recovered & Tsui et al. \\
\hline 82 years/female & Endophthalmitis & Vitreous biopsy & - & Recovered & Reddy et al. \\
\hline 83 years/male & Bacteremia & Blood & $\begin{array}{l}\text { Amikacin, } \\
\text { piperacillin-tazobactam }\end{array}$ & Recovered & Katircioglu et al. \\
\hline 63 years/female & $\begin{array}{l}\text { Catheter related bloodstream } \\
\text { infection in patient on hemodialysis }\end{array}$ & Blood & Vancomycin, ceftriaxone & Dead & Nseir et al. \\
\hline 10 years/male & Bacteremia & Blood & Ciprofloxacin, amikacin & Recovered & Farshad et al. \\
\hline 19 years/female & Bacteremia & Blood & $\begin{array}{l}\text { Ciprofloxacin, vancomycin, } \\
\text { imipenem }\end{array}$ & Recovered & Farshad et al. \\
\hline 51 years/male & Endocarditis & Aortic valve & Ciprofloxacin & Recovered & Arzu Duran et al. \\
\hline 80 years/female & Bacteremia & Blood & Cefazolin and doripenem & Recovered & Orsini Jose et al. \\
\hline 42 years/female & Septic shock & Blood and urine & Ceftazidime and levofloxacin & Recovered & Hyun Jung Kim et al. \\
\hline 65 years/female & Gastroenteritis & Stool & Ciprofloxacin and probiotics & Recovered & Shaika Farooq et al. \\
\hline 46 years/female & Bacteremia & Blood and urine & Gentamicin and imipenem & Recovered & Present case \\
\hline
\end{tabular}

was a female patient. Most of the patient responded well to appropriate antimicrobial therapy. Comamonas species are usually susceptible to aminoglycosides, fluoroquinolones, carbapenems, piperacillin-tazobactam, trimethoprim-sulfamethoxazole, and cephalosporins.

Until 1987, C. testosteroni has only rarely been associated to human infections. In the late 1980s, researchers reported 18 unrelated cases during a research $>3$ years suggesting that this species might be missed if not carefully identified by clinical microbiology laboratories. Although the pathogenic potential of C. testosteroni has not been well studied, it is usually considered a commensal. Because $C$. testosteroni can be isolated from fresh water, it seems likely that wound infection could occur after contact with contaminated water.

Intra-abdominal infections are the most common infections caused by $C$. testosteroni. Bacterial translocation from gastrointestinal tract seems to play an important role in the pathogenesis caused by Comamonas species. ${ }^{[9]}$ The high genetic pliability of Comamonas species, as well as their inherent ability as an environmental pathogen to survive in various ecological niches, makes it an appropriate candidate to cause mild but persistent infections, particularly in an individual with predisposing conditions. In our case, there was no obvious source of infection. The patient did not have central venous catheters, USG abdomen did not show any mass. The patient did not have any immunological deficiency such as malignancy and diabetes mellitus. HIV status was negative. All these factors might have played a role as predisposing factors. In the present case, the patient belongs to rural area so the source of infection might be the contaminated food and water, although this hypothesis cannot be proven. It is only presumed as there was no proven source of infection. 
Polymicrobial bacteremia involving C. testosteroni have been reported in association with Streptococcus parasanguis, Streptococcus agalactiae, Ralstonia pickettii, and Bacteroides fragilis. ${ }^{[10]} \mathrm{C}$. testosteroni is able to survive in the hospital environment for a long time, and majority of infections are community acquired. It is anticipated that nosocomial infection related to this bacterium will increase over time as there is increase in long hospital stay with impaired immune system and long-term use of the intravenous catheter. Most of the laboratories are not well equipped to detect such atypical pathogens that can cause human infection in certain clinical settings. Therefore, automated identification system (VITEK-2) can help to identify such rare isolates. In this case report, we have highlighted an unusual cause of bloodstream infection caused by $C$. testosteroni. Comamonas species should be kept in mind as a rare cause of bacteremia. The main target to report such an unusual case is to sensitize clinicians and clinical microbiologists for bloodstream infections caused by such unusual pathogens. However, more research should be done to understand the pathogenicity, virulence mechanism, and increased antibiotic resistance of $C$. testosteroni.

\section{Declaration of patient consent}

The authors certify that they have obtained all appropriate patient consent forms. In the form the patient(s) has/ have given his/her/their consent for his/her/their images and other clinical information to be reported in the journal. The patients understand that their names and initials will not be published and due efforts will be made to conceal their identity, but anonymity cannot be guaranteed.

\section{Financial support and sponsorship} Nil.

\section{Conflicts of interest}

There are no conflicts of interest.

\section{References}

1. Tsui TL, Tsao SM, Liu KS, Chen TY, Wang YL, Teng YH, et al. Comamonas testosteroni infection in Taiwan: Reported two cases and literature review. J Microbiol Immunol Infect 2011;44:67-71.

2. Orsini J, Tam E, Hauser N, Rajayer S. Polymicrobial bacteremia involving Comamonas testosteroni. Case Rep Med 2014;2014:578127.

3. Kim HJ, Lee Y, Oh K, Choi SH, Sung H, Huh JW. Septic shock due to unusual pathogens, Comamonas testosteroni and Acinetobacter guillouiae in an immune competent patient. Korean J Crit Care Med 2015;30:180-3.

4. Duran A, Abacilar AF, Uyar IS, Akpinar MB, Sahin V, Okur FF, et al. Comamonas testosteroni endocarditis in Turkey: A case report and review of the literature. Int med J Sifa University 2015;2:44-7.

5. Farooq S, Farooq R, Nahvi N. Comamonas testosteroni: Is it still a rare human pathogen? Case Rep Gastroenterol 2017;11:42-7.

6. Reddy AK, Murthy SI, Jalali S, Gopinathan U. Post-operative endophthalmitis due to an unusual pathogen, Comamonas testosteroni. J Med Microbiol 2009;58:374-5.

7. Cooper GR, Staples ED, Iczkowski KA, Clancy CJ. Comamonas (Pseudomonas) testosteroni endocarditis. Cardiovasc Pathol 2005;14:145-9.

8. Nseir W, Khateeb J, Awawdeh M, Ghali M. Catheter-related bacteremia caused by Comamonas testosteroni in a hemodialysis patient. Hemodial Int 2011;15:293-6.

9. Gul M, Ciragil P, Bulbuloglu E, Aral M, Alkis S, Ezberci F, et al. Comamonas testosteroni bacteremia in a patient with perforated acute appendicitis. Short communication. Acta Microbiol Immunol Hung 2007;54:317-21.

10. Opota O, Ney B, Zanetti G, Jaton K, Greub G, Prod'hom G, et al. Bacteremia caused by Comamonas kerstersii in a patient with diverticulosis. J Clin Microbiol 2014;52:1009-12. 